\title{
The Chaotic Monopoly Price Growth Model
}

\author{
Vesna D. Jablanovic \\ University of Belgrade, Belgrade, Serbia
}

\begin{abstract}
Deterministic chaos refers to an irregular or chaotic motion that is generated by nonlinear systems. The chaotic behavior is not to quantum-mechanical-like uncertainty. Chaos theory is used to prove that erratic and chaotic fluctuations can indeed arise in completely deterministic models. Chaotic systems exhibit a sensitive dependence on initial conditions. Seemingly insignificant changes in the initial conditions produce large differences in outcomes. To maximize profit, the monopolist must first determine its costs and the characteristics of market demand. Given this knowledge, the monopoly firm must then decide how much to produce. The monopoly firm can determine price, and the quantity it will sell at that price follows from the market demand curve. The basic aim of this paper is to construct a relatively simple chaotic growth model of the monopoly price that is capable of generating stable equilibria, cycles, or chaos. A key hypothesis of this work is based on the idea that the coefficient, $\pi=\left[\frac{e b}{m(\alpha-1)(e+1)}\right]$ plays a crucial role in explaining local stability of the monopoly price, where, $b$ - the coefficient of the marginal cost function of the monopoly firm, $m$ - the coefficient of the inverse demand function, $e$ - the coefficient of the price elasticity of the monopoly demand, $\alpha$ - the coefficient.

Keywords: monopoly, price, chaos
\end{abstract}

\section{Introduction}

Chaos theory attempts to reveal structure in unpredictable dynamic systems. It is important to construct deterministic, nonlinear economic dynamic models that elucidate irregular, unpredictable economic behavior. Deterministic chaos refers to irregular or chaotic motion that is generated by nonlinear systems evolving according to dynamical laws that uniquely determine the state of the system at all times from the knowledge of the system's previous history. Chaos embodies three important principles: (1) extreme sensitivity to initial conditions; (2) cause and effect are not proportional; and (3) nonlinearity.

Chaos theory can explain effectively unpredictable economic long time behavior arising in a deterministic dynamical system because of sensitivity to initial conditions. A deterministic dynamical system is perfectly predictable given perfect knowledge of the initial condition, and is in practice always predictable in the short term. The key to long-term unpredictability is a property known as sensitivity to (or sensitive dependence on) initial conditions.

Chaos theory started with Lorenz's (1963) discovery of complex dynamics arising from three nonlinear

Vesna D. Jablanovic, Associate Professor of Eonomics, Faculty of Agriculture, University of Belgrade.

Correspondence about this article should be sent to Vesna D. Jablanovic, Vinogradski venac 20, 11000 Belgrade, Serbia. E-mail: vesnajab@ptt.rs. 
differential equations leading to turbulence in the weather system. Li and Yorke (1975) discovered that the simple logistic curve can exibit very complex behavior. Further, May (1976) described chaos in population biology. Chaos theory has been applied in economics by Benhabib and Day (1981, 1982), Day (1982, 1983, 1997), Grandmont (1985), Goodwin (1990), Medio (1993, 1996), Lorenz (1993), Jablanovic (2010, 2011), among many others.

The basic aim of this paper is to provide a relatively simple chaotic the monopoly price growth model that is capable of generating stable equilibria, cycles, or chaos.

\section{A Simple Chaotic Price Growth Model of a Profit-Maximizing Monopoly}

In the model of a profit-maximizing monopoly, take the inverse demand function:

$$
P_{t}=n-m Q_{t}
$$

Where $P$-monopoly price; $Q$-monopoly output; $n, m$-coefficients of the inverse demand function.

Further, suppose the quadratic marginal-cost function for a monopoly is:

$$
M C_{t}=a+b Q_{t}+c Q_{t}^{2}
$$

$M C$ - marginal cost; $Q$ —-monopoly output ; $a, b, c$-coefficients of the quadratic marginal-cost function.

Marginal revenue is:

$$
M R_{t}=P_{t}\left[1+\left(\frac{1}{e}\right)\right]
$$

$M R$ - marginal revenue; $P$ - monopoly price; $e$ - the coefficient of the price elasticity of demand.

A monopoly firm maximizes profit by producing the quantity at which marginal revenue equals marginal cost. Thus the profit-maximizing condition is that:

Further,

$$
M R_{t}=M C_{t}
$$

$$
\begin{gathered}
M C_{t+1}=M C_{t}+\Delta M C \\
M C_{t+1}=M C_{t}+\alpha M C_{t+1} \\
(1-\alpha) M C_{t+1}=M C_{t}
\end{gathered}
$$

Thus, the chaotic model of the profit-maximizing monopoly is presented by the equations (1)-(4) and (7). Where: $Q$ —output of the monopoly firm; $M C$ - marginal cost; $M R$ - marginal revenue; $P$-monopoly price; $e$ - the coefficient of the price elasticity of demand; $n, m$ - coefficients of the inverse demand function; $a, b$, $c$ - coefficients of the quadratic marginal-cost function.

Firstly, it is supposed that $a=0$ and $n=0$.

By substitution one derives:

$$
P_{t+1}=\frac{e b}{m(\alpha-1)(e+1)} P_{t}-\frac{e c}{m^{2}(\alpha-1)(e+1)} P_{t}^{2}
$$

Further, it is assumed that the monopoly price is restricted by its maximal value in its time series. This premise requires a modification of the growth law. Now, the monopoly price growth rate depends on the current size of the monopoly price, $P$, relative to its maximal size in its time series $P^{m}$. We introduce $p$ as $p=P / P^{m}$. Thus, $p$ ranges between 0 and 1 . Again we index $p$ by $t$, i.e., write $p_{t}$ to refer to the size at time steps $t=0,1,2,3, \ldots$. Now, growth rate of the monopoly price is measured as: 


$$
p_{t+1}=\frac{e b}{m(\alpha-1)(e+1)} p_{t}-\frac{e c}{m^{2}(\alpha-1)(e+1)} p_{t}{ }^{2}
$$

This model given by equation (9) is called the logistic model. For most choices of $b, c, m$, and $e$, there is no explicit solution for equation (9). Namely, knowing $b, c, m$, and $e$ and measuring $p_{0}$ would not sufficient to predict $p_{t}$ for any point in time, as it was previously possible. This is at the heart of the presence of chaos in deterministic feedback processes. Lorenz (1963) discovered this effect-the lack of predictability in deterministic systems. Sensitive dependence on initial conditions is one of the central ingredients of what is called deterministic chaos.

This kind of difference equation (9) can lead to a very interesting dynamic behavior, such as cycles that repeat themselves every two or more periods, and even chaos, in which there is no apparent regularity in the behavior of $p_{t}$. This difference in equation (9) will possess a chaotic region. Two properties of the chaotic solution are important: firstly, given a starting point $p_{0}$ the solution is highly sensitive to variations of the parameters $b, c, m$, and $e$; secondly, given the parameters $b, c, m$, and $e$ the solution is highly sensitive to variations of the initial point $p_{0}$. In both cases the two solutions are for the first few periods rather close to each other, but later on they behave in a chaotic manner.

\section{Logistic Equation}

The logistic map is often cited as an example of how complex, chaotic behavior can arise from very simple non-linear dynamical equations. The logistic model was originally introduced as a demographic model by Pierre François Verhulst. It is possible to show that iteration process for the logistic equation:

$$
z_{t+1}=\pi z_{t}\left(1-z_{t}\right), \pi \in[0,4], z_{t} \in[0,1]
$$

is equivalent to the iteration of growth model (9) when we use the following identification:

$$
z_{t}=\frac{e c}{e b m} p_{t}
$$

and

$$
\pi=\left[\frac{e b}{m(\alpha-1)(e+1)}\right]
$$

Using equation (9) and equation (11) we obtain:

$$
\begin{aligned}
z_{t+1}=\left(\frac{e c}{e b m}\right) p_{t+1} & =\left(\frac{e c}{e b m}\right)\left[\left(\frac{e b}{m(\alpha-1)(e+1)}\right) p_{t}-\left(\frac{e c}{m^{2}(\alpha-1)(e+1)}\right) p_{t}{ }^{2}\right] \\
& =\left(\frac{e c}{m^{2}(\alpha-1)(\mathrm{e}+1)}\right) p_{t}-\left(\frac{\mathrm{e}^{2} \mathrm{c}^{2}}{\mathrm{e} \mathrm{bm}^{3}(\alpha-1)(\mathrm{e}+1)}\right) \mathrm{p}_{\mathrm{t}}^{2}
\end{aligned}
$$

On the other hand, using equations (10)-(12) we obtain:

$$
\begin{aligned}
z_{t+1}=\pi z_{t}\left(1-z_{t}\right) & =\left(\frac{e b}{m(\alpha-1)(e+1)}\right)\left(\frac{e c}{e b m}\right) p_{t}\left[1-\left(\frac{e c}{e b m}\right) p_{t}\right] \\
= & \left(\frac{e c}{m^{2}(\alpha-1)(e+1)}\right) p_{t}-\left(\frac{e^{2} c^{2}}{e b m^{3}(\alpha-1)(e+1)}\right) p_{t}{ }^{2}
\end{aligned}
$$


Thus, we have that iterating $p_{t+1}=\frac{e b}{m(\alpha-1)(e+1)} p_{t}-\frac{e c}{m^{2}(\alpha-1)(e+1)} p_{t}^{2}$ is really the same as iterating $z_{t+1}=\pi z_{t}\left(1-z_{t}\right)$ using $z_{t}=\frac{e c}{e b m} p_{t}$ and $\pi=\left[\frac{e b}{m(\alpha-1)(e+1)}\right]$.

It is important because the dynamic properties of the logistic equation (10) have been widely analyzed (Li \& Yorke, 1975; May, 1976).

It is obtained that:

(1) For parameter values $0<\pi<1$ all solutions will converge to $z=0$;

(2) For $1<\pi<3.57$, there exist fixed points the number of which depends on $\pi$,

(3) For $1<\pi<2$, all solutions monotnically increase to $z=(\pi-1) / \pi$,

(4) For $2<\pi<3$, fluctuations will converge to $\mathrm{z}=(\pi-1) / \pi$,

(5) For $3<\pi<4$, all solutions will continously fluctuate;

(6) For $3.57<\pi<4$, the solution become "chaotic" wihch means that there exist a totally aperiodic solution or periodic solutions with a very large, complicated period. This means that the path of $z_{t}$ fluctuates in an apparently random fashion over time, not settling down into any regular pattern whatsoever.

\section{Conclusion}

This paper suggests conclusion for the use of the simple chaotic model of a profit-maximizing monopoly in predicting the fluctuations of the monopoly price. The model (9) has to rely on specified parameters $b, c, m$, and $e$, and initial value of the monopoly price, $p_{0}$. But even slight deviations from the values of parameters parameters $b, c, m$, and $e$ and initial value of the monopoly price, show the difficulty of predicting a long-term behavior of the monopoly price, $p_{0}$.

A key hypothesis of this work is based on the idea that the coefficient plays a crucial role in explaining local stability of the monopoly price:

$$
\pi=\left[\frac{e b}{m(\alpha-1)(e+1)}\right]
$$

where $b$ is the coefficient of the marginal cost function of the monopoly firm, $m$ is the coefficient of the inverse demand function, $e$ is the coefficient of the price elasticity of monopoly's demand, $\alpha$ is the coefficient.

The quadratic form of the marginal cost function of the monopoly firm is important ingredient of the presented chaotic monopoly price growth model (9).

\section{References}

Benhabib, J., \& Day, R. H. (1981). Rational choice and erratic behaviour. Review of Economic Studies, 48, 459-471.

Benhabib, J., \& Day, R. H. (1982). Characterization of erratic dynamics in the overlapping generation model. Journal of Economic Dynamics and Control, 4, 37-55.

Benhabib, J., \& Nishimura, K. (1985). Competitive equilibrium cycles. Journal of Economic Theory, 35, 284-306.

Day, R. H. (1982). Irregular growth cycles. American Economic Review, 72, 406-414.

Day, R. H. (1983). The emergence of chaos from classica economic growth. Quarterly Journal of Economics, 98, $200-213$.

Day, R. H. (1997). Complex economic dynamics volume I: An introduction to dynamical systems and market mechanism. In Discrete Dynamics in Nature and Society (pp. 177-178), 1. MIT Press.

Gandolfo, G. (2009). Economic dynamics (4th ed.). Berlin: Springer-Verlag. 
Goodwin, R. M. (1990). Chaotic economic dynamics. Oxford: Clarendon Press.

Grandmont, J. M. (1985). On enodgenous competitive business cycles. Econometrica, 53, 994-1045.

Jablanović, V. (2010). Chaotic population growth. Belgrade: Cigoja.

Jablanović, V. (2011). The chaotic saving growth model: G 7. Chinese Business Review, 10(5), 317-327.

Li, T., \& Yorke, J. (1975). Period three implies chaos. American Mathematical Monthly, 8, 985-992.

Lorenz, E. N. (1963). Deterministic nonperiodic flow. Journal of Atmospheric Sciences, 20, 130-141.

Lorenz, H. W. (1993). Nonlinear dynamical economics and chaotic motion (2nd ed.). Heidelberg: Springer-Verlag.

May, R. M. (1976). Mathematical models with very complicated dynamics. Nature, 261, 459-467.

Medio, A. (1993). Chaotic dynamics: Theory and applications to economics. Cambridge: Cambridge University Press.

Medio, A. (1996). Chaotic dynamics. Theory and applications to economics. De Economist, 144(4), 695-698.

Peitgen, H. O., Jürgens, H., \& Saupe, D. (1992). Chaos and fractals-new frontiers of science. New York: Springer-Verlag.

Rössler, O. E. (1976). An equation for continuous chaos. Physics Letters A, 57, 397-398.

Tu, P. N. V. (1994). Dynamical systems. Verlag: Springer. 